\title{
Videokonferenzunterstützte Projektarbeit und Präsentation - Simulation eines Fertigungsbereiches
}

\author{
Nadine Röhtz, Peter Wasser
}

\section{Eingangsbemerkungen}

Nach der erfolgreichen Umsetzung des Projektes „Videokonferenzunterstützte Vorlesung " (siehe Hentschel/Wasser, Wissenschaftliche Beiträge 1/1997) galt es, neue Einsatzmöglichkeiten der Videokonferenztechnik zu finden und an der Technischen Fachhochschule Wildau umzusetzen.

In einer modernen Informationsgesellschaft ist es notwendig, neue technische Möglichkeiten zu erschließen und in die Arbeit zu integrieren. In Anbetracht logistischer Gesichtspunkte und den sehr guten Erfahrungen bei der Arbeit mit Videokonferenztechnik wollten wir aktiv dem vermehrten Verkehrsaufkommen auf deutschen Straßen entgegenwirken. Ansätze dafür lieferte in unserem Fall die Kooperation mit verschiedenen Industrieunternehmen. Zur Abstimmung und Realisierung der Projektarbeit war es bisher notwendig, umfangreiche Dienstreisen durchzufuihren. Den dabei entstehenden hohen Kosten- und Zeitaufwand so weit wie möglich durch den Einsatz neuer Informations- und Kommunikationstechnologien zu minimieren, sollte unser Ziel sein.

Beispiele aus Großunternehmen, die weltweit miteinander kooperieren und kommunizieren inspirierten uns zusätzlich auch auf diesem Gebiet tätig zu werden, und neue Technologien in unsere Projektarbeit zu integrieren.

Aus dem Einsatz der Kommunikationstechnologie Videokonferenz ergeben sich Vorteile, die einen erheblichen Beitrag zur Umsetzung unseres Vorhabens leisten und die einen nicht unbedeutenden Wettbewerbsvorteil darstellen können:

- sehr schneller Informationsfluß

kein Warten auf herkömmliche Konferenzen, Probleme werden dann gelöst, wenn sie auftreten

- hohe Flexibilität und Spontaneität jederzeit Verbindung möglich

- schnelle Verfügbarkeit von Experten jederzeit Verbindung möglich

- hohe Zeiteinsparung durch Wegfall von Dienstreisen Wartezeit bei Reiseantritt bzw. Fahrzeit entfällt

- Kosteneinsparung durch Wegfall der Dienstreisen Hotelkosten, Fahrkosten, Arbeitsabwesenheit

- Beitrag zur Ökologie durch Vermeidung von Dienstreisen

Vermindertes Verkehrsaufkommen

- geringe Fehler beim Informationsaustausch durch Visualisierung der Gesprächsinhalte

Mimik und Gestik ist erkennbar, Erklärungen einfacher
Die überzeugenden Vorteile veranlaßten uns dazu, eine erstes Projekt mittels Videokonferenz abzuarbeiten. Die Lösungsansätze, Erkenntnisse und Probleme möchten wir im folgenden darstellen.

\section{Zielstellung}

Anhand des Projektes „Entwurf und Simulation eines technologischen Prozesses - Stahlkiesstrahlen“ sollte erstmals der Versuch unternommen werden, Videokonferenztechnik in Projektarbeit zwischen der Technischen Fachhochschule Wildau und einem externen Unternehmen einzubinden.

Ein Bestandteil des Projektes war es, eine Materialflußsimulation für einen Produktionsprozeß zu erstellen. Als Grundlage dienten die ermittelten Einsatzzeiten der IstAnalyse. Die daraus entwickelte Simulation sollte durch die Änderung möglicher Parameter optimiert werden. Zur effektiven Optimierung sollte dies gemeinsam mit den Verantwortlichen des Unternehmens geschehen. So kann zum einen der Projektbearbeiter und Entwickler der Simulation seine Vorschläge einbringen, zum anderen die Mitarbeiter des Unternehmen diese Vorschläge bewerten bzw. selbst Verbesserungspotentiale definieren.

Zur Realisierung dieser Vorgehensweise dienten vor allem die integrierten Funktionen der Videokonferenzsoftware, wie Applikation Sharing. Applikation Sharing umfaßt die gemeinsame Nutzung von beliebigen Windows-Anwendungen zwischen den Teilnehmern. Die Dokumente sind während der Bearbeitung bei den Teilnehmern gleichzeitig zu sehen und können gemeinsam bearbeitet werden. Alle Funktionen des Programms stehen zur Verfuigung und Änderungen werden sofort sichtbar. Dabei ist es ausreichend, wenn das Programm auf nur einem Rechner installiert ist und das Dokument auf einem Rechner geöffnet wird.

Zur Simulation des Prozesses wurde das Simulationsprogramm Simpro vom Simulations- und Dienstleistungszentrum Dortmund (SDZ) genutzt. Das Programm dient zur Simulation von Materialflußsystemen und Fertigungsabläufen. Simpro ist ein objektorientiertes Programm. Die Objekte stellen zum einen Elemente dar, in den die Bearbeitungsprozesse ablaufen, aber auch Teile die während der Prozesse transformiert werden. Die Elemente lassen sich darstellen und erlauben bestimmte Operationen. Objekte mit gleichen Eigenschaften werden in Klassen zusammengefaßt, die als Ober- 
und Unterklassen hierarchisch aufgebaut sind. Klassen mit unterschiedlichen Eigenschaften werden zu Bibliotheken zusammengefaßt. Eine besondere Form davon ist der Benutzerbehälter, ist der Arbeitsbereich des Anwenders.

Es gibt verschiedene, bereits vordefinierte Bausteintypen für Bearbeitung, Demontage, Montage usw., welche sich in einer Standardbibliothek befinden. Auch Transportmittel, Werker und Strecken lassen sich darstellen. Es ist außerdem möglich, selbst Bausteine zu erstellen. Die für den betrachteten Prozeß relevanten Daten werden in bereits vordefinierte Parametermasken der Bausteine eingesetzt. Für den Betrachter wird die Simulation eines Prozesses durch eine Animation angezeigt. Um den Prozeß zu optimieren, können einzelne Parameter geändert werden. Für die Simulation kann ein Zeitabschnitt beliebiger Länge gewählt werden. Durch das Auswerten von Statistiken, Tabellen und Diagrammen lassen sich Schwachstellen bzw. Engpässe erkennen.

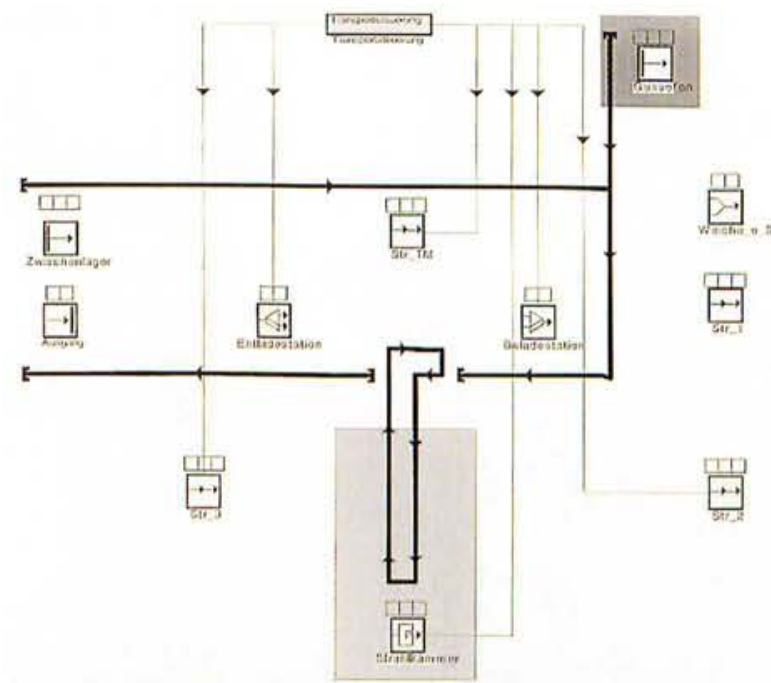

Abb. 1: Layout der Simulation im Programm Simpro (Bausteinanordnung)

\section{Ablauf des Projektes}

Ursprünglich war geplant das Projekt so zu gestalten, daß neben der Videokonferenz das Programm Simpro mittels Applikation Sharing genutzt wird. Simpro läuft unter dem Betriebssystem Windows NT. Somit war die Realisierung der Aufgabe mit einem Rechner pro Endteilnehmer nicht möglich, da es bis Ende 1997 keine Videokonferenzsysteme für Windows NT gab. Nach Auskunft von MVC (Mobile VideoCommumication Deutschland $\mathrm{GmbH}$, Vertriebspartner PictureTel) gibt es ein solches System ab dem 2. Quartal 1998.

Daraus entstand die Überlegung, vorläufig zwei Rechner pro Endstelle zu nutzen. Auf dem einen Rechner ist die Software des Videokonferenzsystems installiert. Dieser dient zur visuellen Kommunikation und zur Sprachübertragung während der Konferenz. Auf einem zweiten, unmittelbar danebenstehenden Rechner ist eine Software zur gemeinsamen Nutzung der Simproanwendung vorhanden. Mit Hilfe beider PC's kann nun sowohl das Projekt gemeinsam besprochen werden als auch Parameteränderungen innerhalb der Simulation von beiden Seiten ausgeführt werden. Ein zusätzlicher Vorteil dieser Variante ist, daß der Zugriff unabhängig davon erfolgen kann, ob beide Endteilnehmer am Platz sind. Über die Funktion Remote Control ist es möglich, rund um die Uhr Zugriff auf die Gegenstellen zu erhalten.

Die Variante erhält einen besonderen Stellenwert, bei internationalen Projekten dezentral organisierter Unternehmen oder Projektgruppen. Diese Art der Projektbearbeitung wird derzeit bei Mercedes Benz realisiert. Die Konstruktionsabteilung des Unternehmens befindet sich in den USA und die Tochtergesellschaften können, ohne Berïcksichtigung der Zeitverschiebung miteinander direkt kooperieren.

In einem Testlauf an der Hochschule konnten gute Ergebnisse für die Nutzung der Technik erzielt werden. Dabei wurde das Simulationsprogramm mit Hilfe der Kommunikationssoftware PC-Anywhere gemeinsam von den Endstellen genutzt. Verbunden waren beide Endstellen über das interne Netzwerk. Daher erfolgte die Bearbeitung mit einem erheblichen Geschwindigkeitsvorteil gegenüber der Übertragung mittels ISDN. Für die sinnvolle Anwendung des Applikation Sharing über ein Videokonferenzsysteme sind daher mindestens 3 ISDNLeitungen erforderlich.

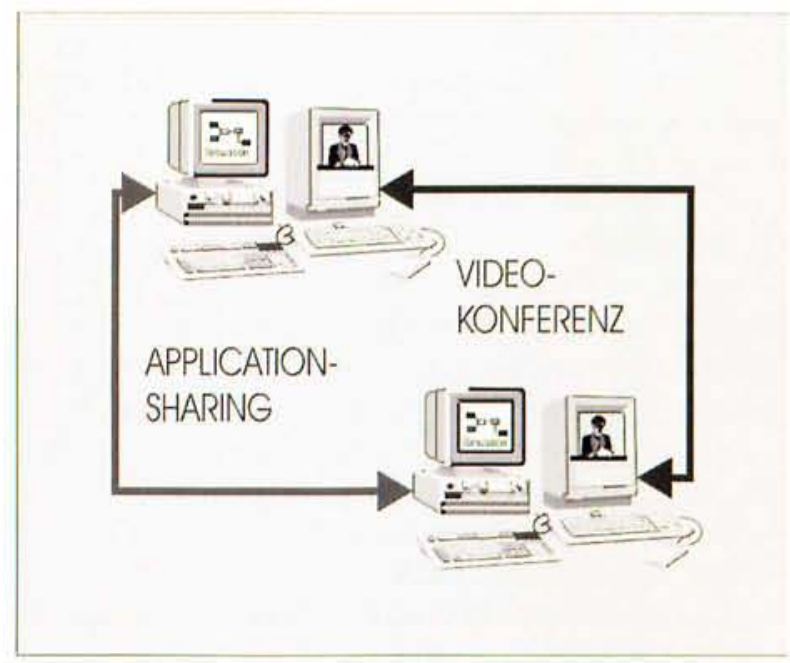

Abb. 2: Schematischer Aufbau der Kommunikation

\section{Abschlußbemerkungen}

Zur Verwirklichung der Zielsetzung des Projektes mit nur einem Rechner pro Endstelle, sind die Tests der Videokonferenzsysteme für das Betriebssystem Windows NT abzuwarten. Es ist allerdings zu bedenken, daß diese Systeme neu und wahrscheinlich unausgereift, sowie sehr teuer sind. Fraglich ist ebenfalls, ob und in welchem Maße Applikation Sharing möglich sein wird, da Simpro auf einer Unix-Oberfläche über dem Betriebssystem Windows NT arbeitet.

Bei dem Einsatz von Videokonferenzsystemen ist vor allem zu beachten, daß die genutzten Systeme kompa- 
tibel zueinander sind. Obwohl alle Systeme die Standards T.120 (Übertragung Multimediadaten) und H.320 (Video/Audiosignale) unterstuitzen, wurden in Versuchen mit Systemen verschiedener Hersteller unterschiedliche Ergebnisse festgestellt. In Bereich Applikation Sharing ergab sich nur dann ein sinnvoller Einsatz, wenn beide Endstellen mit Systemen des gleichen Herstellers kommunizierten.

Die Kommunikationsmöglichkeit mittels Videokonferenz wird in Zukunft immer mehr an Bedeutung gewinnen. In Anlehnung an die genannten Vorteile des Einsatzes solcher Systeme, läßt sich bei den Unternehmen einer steigende Akzeptanz gegenuiber der Anwendung der Technologie feststellen. Dies gilt sowohl für die externe als auch für die interne Kommunikation der Unternehmen.

Da die Programme die uiber das Betriebssystem Windows 95 laufen, vollständig unterstiutzt werden, lassen sich z. B. die Aufwendungen für Dienstreisen zur Zwischenpräsentation von Projektergebnissen deutlich reduzieren. Auch der Austausch von Daten und Informationen läßt sich durch die Standardfunktionen von Videokonferenzsoftware einfach und schnell realisieren. Trotzdem wird der persönliche Kontakt zu den Projektpartner weiterhin nötig sein.

Sinnvoll eingesetzte Videokonferenzen sind eine hervorragende Möglichkeit den Verkehr und die damit verbundenen Kosten, besonders aber die zunehmende Umweltverschmutzung, zu vermindern.

\section{Ausblick}

Mit der Einführung des Internationalen Studienganges Logistik im Wintersemester 1998/99 ergeben sich Möglichkeiten, das Projekt „Videokonferenzunterstützte Vorlesung" weiterzuführen. Zur Kooperation zwischen den Partnerhochschulen

- Technischen Fachhochschule Wildau

- Technische Universität Poznan (Polen)

- Hogeschool van Arnheim en Nijmegen (Holland)

- Müszaki Föiskola „K. Kalman“ Budapest (Ungarn)

- Staatliche Technische Universität Jaroslawel (Rußland) läßt sich die Videokonferenztechnik sinnvoll einsetzen. Dabei ist geplant, die Vorlesungen gleicher Fächer für alle Hochschulen gleichzeitig (z. B. in englischer Sprache) zu halten. Auch der Einsatz von Experten zu bestimmten Themen aus den verschiedenen Ländern läßt sich einfach und schnell realisieren. Dazu wird es allerdings notwendig diese Videokonferenzen genauestens zu planen. Es gibt es zwei verschiedene Lösungsansätze. Es ist möglich die Konferenzen als Point-to-Multipoint - Konferenz zu gestalten. Dabei fungiert die Hochschule an der die Vorlesung stattfindet als „Dozent“. Alle anderen Hochschulen sind Zuhörer und können nur mit „Dozent" direkt kommunizieren. Dadurch kann es häufig zu Überschneidungen bei Fragenstellungen der einzelnen Beteiligten kommen, da sich diese weder sehen noch hören können.

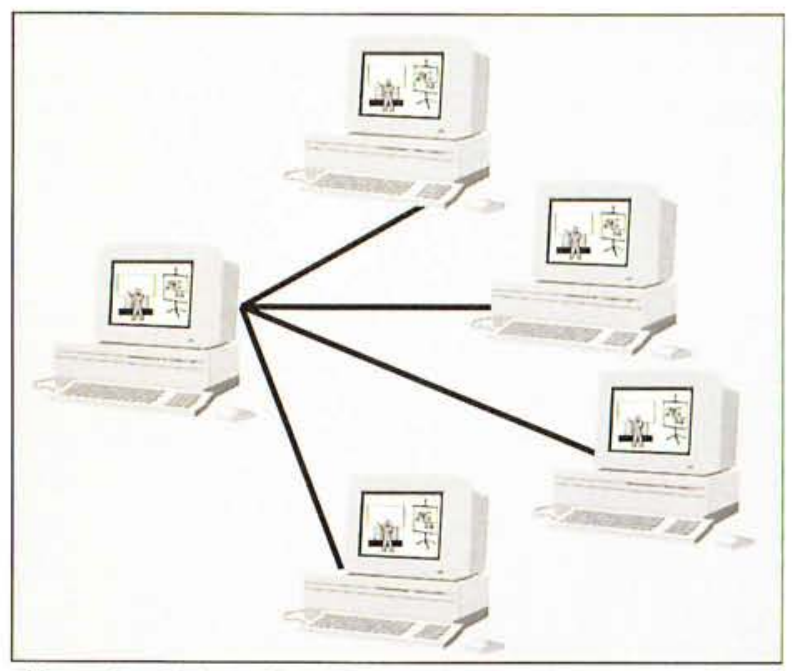

Abb. 3: Schematischer Aufbau Point-to-Multipoint-Konferenz

Die zweite Möglichkeit ist eine Multipoint-Konferenz. Dabei sind während der Videokonferenzschaltung alle beteiligten Hochschulen gleichwertig und können direkt miteinander in Verbindung treten. Die Steuerung erfolgt über eine Multi-Conferencing-Unit.

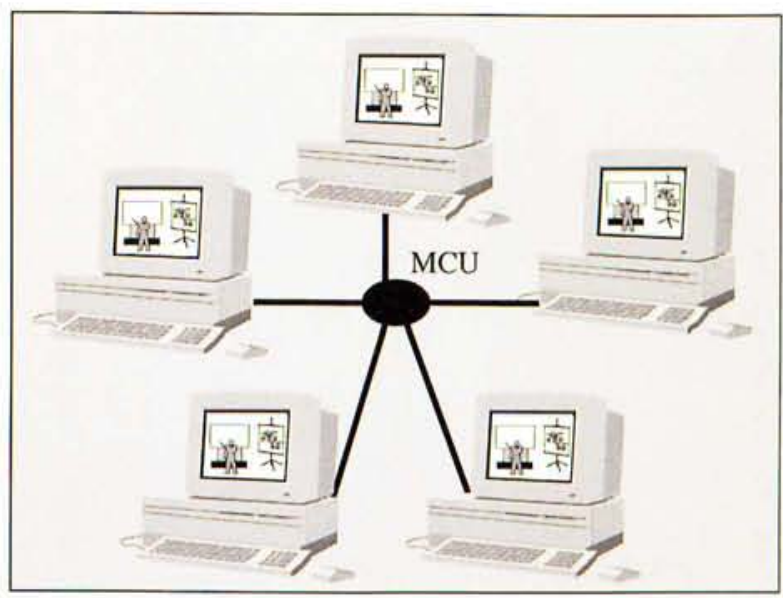

Abb. 4: Schematischer Aufbau Multipoint-Konferenz

Während der Vorbereitungsphase für die Videokonferenz ist genau zu definieren wer, wann das Wort ergreift und wie lange gesprochen wird. Zur Gewährleistung der Spontaneität sind gewisse Zeiträume für Fragen der Studenten einzuplanen. Vorteilhaft ist es, vorab eine Person als Moderator zur Koordination zu bestimmen. Wir sind sicher, daß diese Art der Vorlesung einen nicht unerheblichen Anteil an der Attraktivität des Internationalen Studienganges Logistik haben wird.

\section{Verfasser}

Nadine Röhtz (Studentin)

Technischen Fachhochschule Wildau

Tel. (0 33 75) 508-924

\section{Dipl.-Ing. Peter Wasser}

Technischen Fachhochschule Wildau

Fachbereich Ingenieurwesen/Wirtschaftsingenieurwesen Tel. (0 33 75) 508-925 